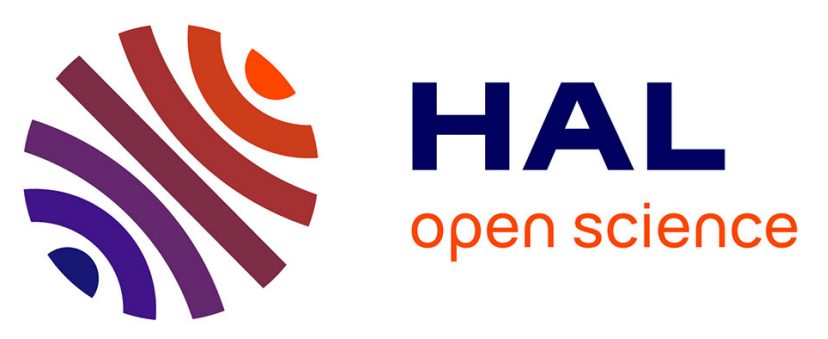

\title{
Multi-messenger astronomy
}

M. Davier

\section{To cite this version:}

M. Davier. Multi-messenger astronomy. 21st International Conference on Neutrino Physics and Astrophysics (Neutrino 2004), Jun 2004, Paris, France. pp.395-406, 10.1016/j.nuclphysbps.2005.01.136 . in2p3-00024232

\section{HAL Id: in2p3-00024232 https://hal.in2p3.fr/in2p3-00024232}

Submitted on 9 Jun 2005

HAL is a multi-disciplinary open access archive for the deposit and dissemination of scientific research documents, whether they are published or not. The documents may come from teaching and research institutions in France or abroad, or from public or private research centers.
L'archive ouverte pluridisciplinaire HAL, est destinée au dépôt et à la diffusion de documents scientifiques de niveau recherche, publiés ou non, émanant des établissements d'enseignement et de recherche français ou étrangers, des laboratoires publics ou privés. 


\section{Multi-messenger Astronomy}

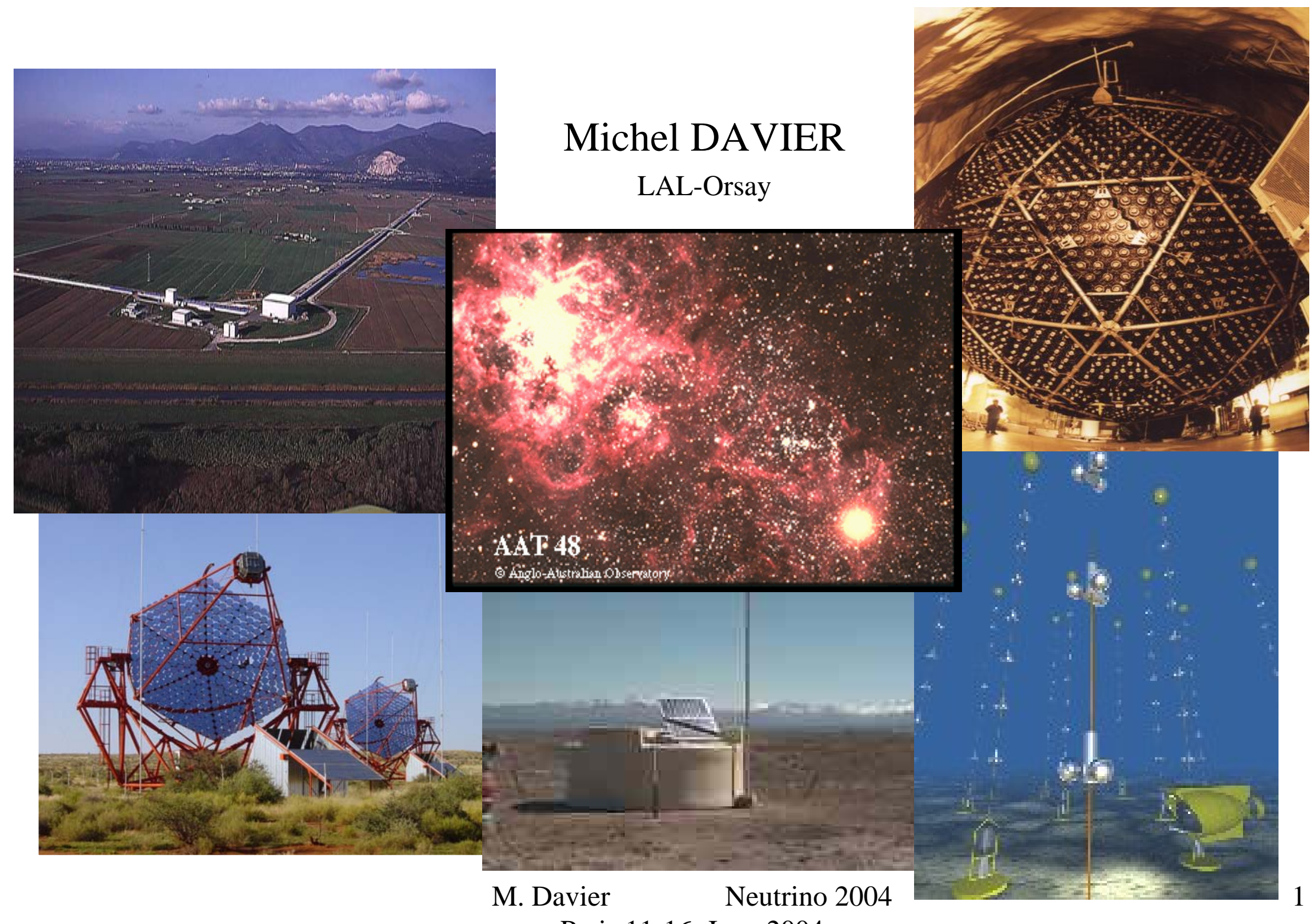

Paris 11-16 June 2004 


\section{General Remarks}

- A vast subject and a very active field

- Multi-messengers:

photons (radio, IR, visible, $\mathrm{X}$ - and $\boldsymbol{\gamma}$-rays)

protons and nuclei

neutrinos

a new comer: gravitational waves

- The Universe looks very different with different probes

- However: important to observe the same events

- Very selective review (focus on interplay) 


\section{Outline}

- UHE Cosmic Rays

- $\gamma$-ray Bursts

- Investigating Dark Matter with $\gamma$-rays

- GW signals : the next galactic SN (a generic case) 


\section{UHE Cosmic Rays}

- Energy spectrum extends to $\sim 10^{20} \mathrm{eV}$

- Shoulder 5. $10^{19} \mathrm{eV}$

- Big questions:

AGASA, Fly's Eye, Yakutsk, HiRes

Problem: energy scale

- Where are the accelerators ? How do they work?

- Is the GZK cutoff seen ?

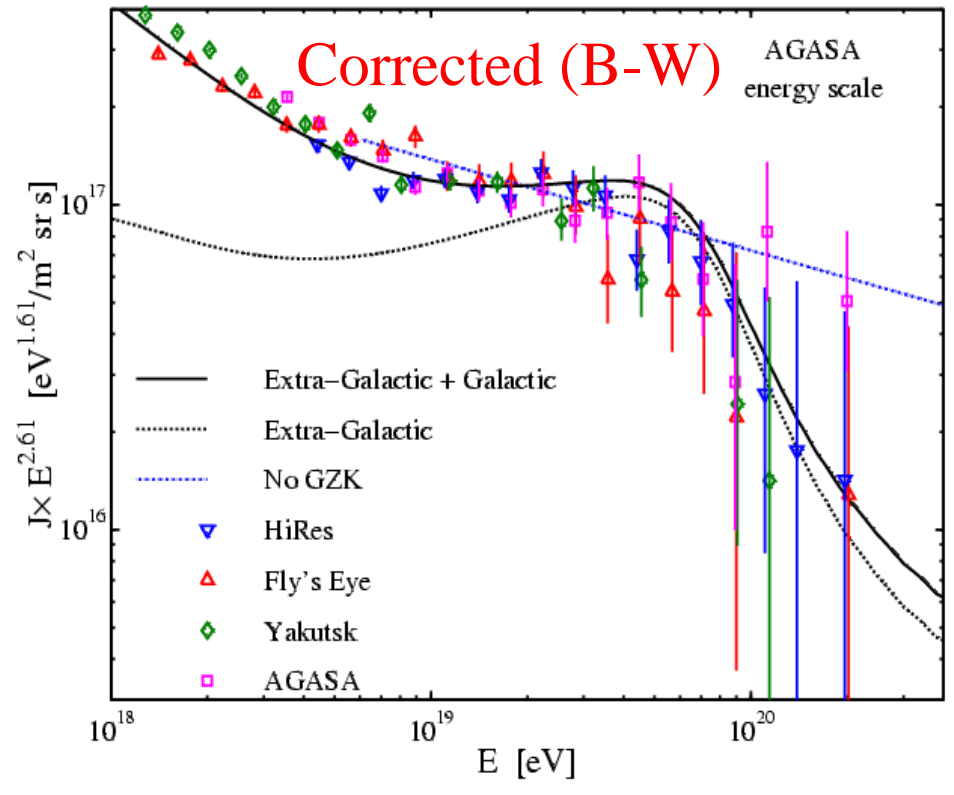

M. Davier proton interactions with CMB photons energy loss distance much reduced $\begin{array}{rr}10 \mathrm{Mpc} & 10^{20} \mathrm{eV} \\ 1 \mathrm{Gpc} & 0.510^{20} \mathrm{eV}\end{array}$

evidence for GZK? (Bahcall-Waxman 03)

Auger expt should settle this point expect $\sim 30$ evts/yr above $10^{20} \mathrm{eV}$ 


\section{GRB : Facts and Interpretation}

- Short variable $\gamma$-ray bursts $\quad 0.01-100 \mathrm{~s} \quad 0.1-1 \mathrm{MeV}$

- Isotropic distribution (BATSE)

- X-ray afterglow (BeppoSAX) $\Rightarrow$ optical and radio afterglows

- Beautiful exemple of multi-wavelength approach (same messenger!)

$\Rightarrow$ Sources at cosmological distances

$\Rightarrow$ Enormous energy release $\sim 10^{53}$ erg + beaming

- Strong support for fireball model (review Piran 00)

- energy source: accretion on a newly formed compact object

- relativistic plasma jet flow

- electron acceleration by shocks

- $\gamma$-rays from synchrotron radiation

- afterglows when jet impacts on surrounding medium

- still many open questions 


\section{GRB : Connections}

- can UHE Cosmic Rays be explained by GRB's ?

Waxman 95, Pietri 95

- relativistic plasma jet can also accelerate protons to $\sim 10^{20} \mathrm{eV}$ Milgrom-Usov 95

- constraints on jet similar for p acceleration and $\gamma$ emission (although indep.)

- energy generation rates similar

- HE neutrinos are expected

- accelerated p interact with fireball photons and produce pions

- $v_{\mu}$ from charged $\pi \quad \Rightarrow v_{\mu}, v_{\tau}$ on Earth $\quad \sim \mathrm{E}_{v}{ }^{-2}$

- expect 20 evts/yr in a $1 \mathrm{~km} 3$ detector up to $10^{16} \mathrm{eV}$ (Waxman-Bahcall 01)

- correlated in time and direction with GRB

- central engine also emits GW (compact object, relativistic motion)

- scenarios to get BH+accretion disk : NS-NS, NS-BH mergers, failed SN

- 'canonical' GW sources (inspiral $\rightarrow$ merger, collapse)

- LIGO-Virgo only sensitive to $30 \mathrm{Mpc}$, advanced LIGO-Virgo to $400 \mathrm{Mpc}$

- $\mathrm{BH}$ ringdown has a distinct signature (normal modes, damped sine GW) 


\section{$\gamma$-ray signatures of Dark Matter (1) \\ Extragalactic $\gamma$-ray background and heavy DM}
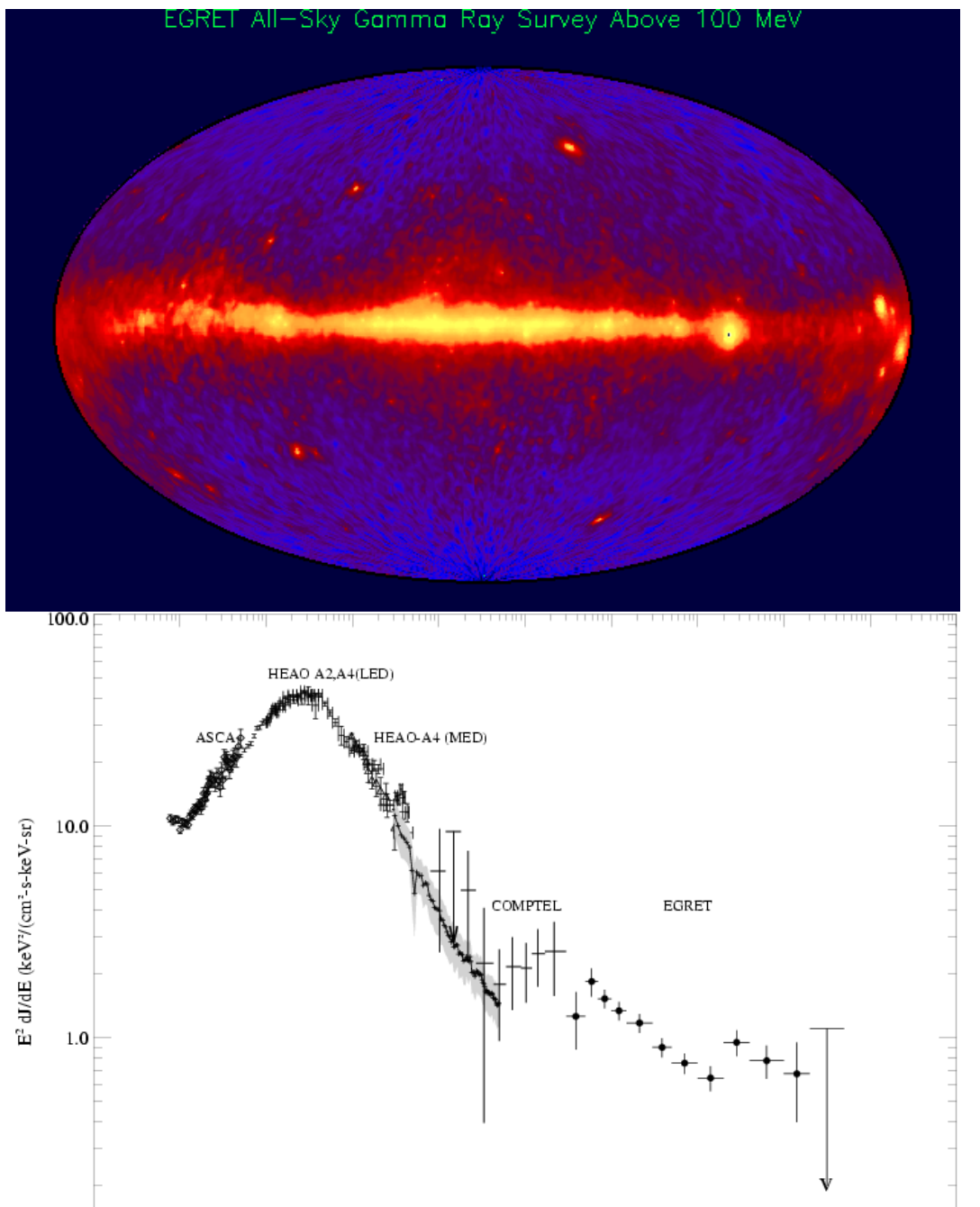

M. Davier
Space Telescopes: EGRET $\rightarrow$ GLAST

$$
30 \mathrm{MeV}-10 \mathrm{GeV}
$$

extragalactic component difficult to determine (isotropy not enough, need model of Galactic background, not firmly establihed) Strong 04 superposition of all unresolved sources (AGN)

? could the HE component result from selfannihilating DM particles (such as SUSY LSP) Elsässer-Mannheim 04 : possibly substantial contribution if mass $=0.5-1 \mathrm{TeV}$, very sensitive to the DM distribution in the Universe more conventional models work (Strong 04a) 


\section{$\gamma$-ray signatures of Dark Matter (3) \\ $511 \mathrm{keV}$ line from the Galactic bulge and light DM}

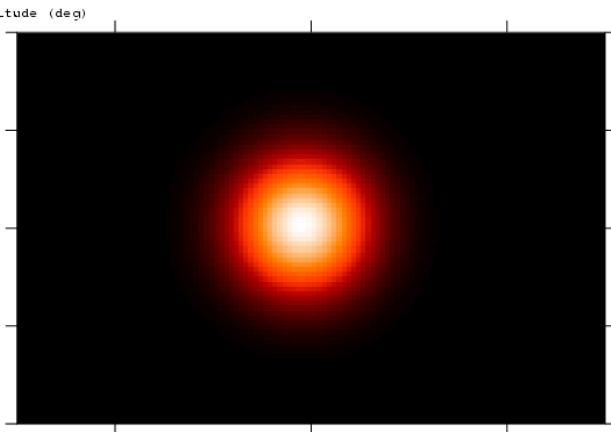

Los git ude (deg)

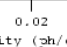

Clear observation by SPI/INTEGRAL of a signal from $\mathrm{e}^{+} \mathrm{e}^{-}$ annihilation at rest in an angular range compatible with the galactic bulge, inconsistent with a single point source

What is the source of positrons ?

'standard' explanation: SN Ia with $\beta^{+}$radioactivity of produced nuclei, but rate appears to be too small (Schanne 04)

Cassé 04, Fayet 04 : light DM particles

$\varphi \operatorname{spin} 1 / 2$ or $0 \quad \mathrm{~m}_{\varphi} \sim \mathrm{O}(1 \mathrm{MeV})$

coupled to a light vector boson $\mathrm{U}$

$\mathrm{m}_{\mathrm{U}} \sim 1-100 \mathrm{MeV}$ (lower range favoured)

$\varphi \bar{\varphi} \rightarrow \mathrm{U} \rightarrow \mathrm{e}^{+} \mathrm{e}^{-}$

astrophysical tests proposed

severely constrained by particle physics

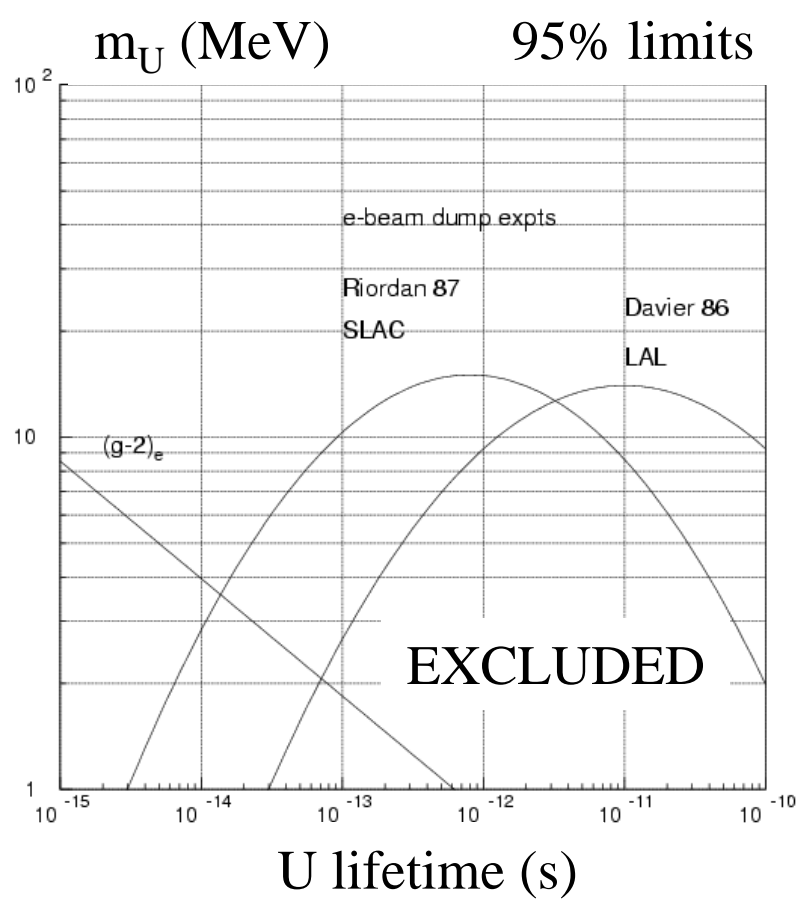




\section{Gravity Wave Detectors}

GW : quadrupolar deformation of space-time metrics amplitude $\mathrm{h}=\Delta \mathrm{L} / \mathrm{L} \quad \Rightarrow$ interferometric detection well suited

Large interferometric antennas coming into operation:

TAMA (Japan), LIGO-Hanford/Livingston (US),

GEO (Germany-UK), Virgo (France-Italy)

Best Strain Sensitivities for the LIGO Interferometers

Comparisons among S1, S2, S3 LIGO-G030548-01-E

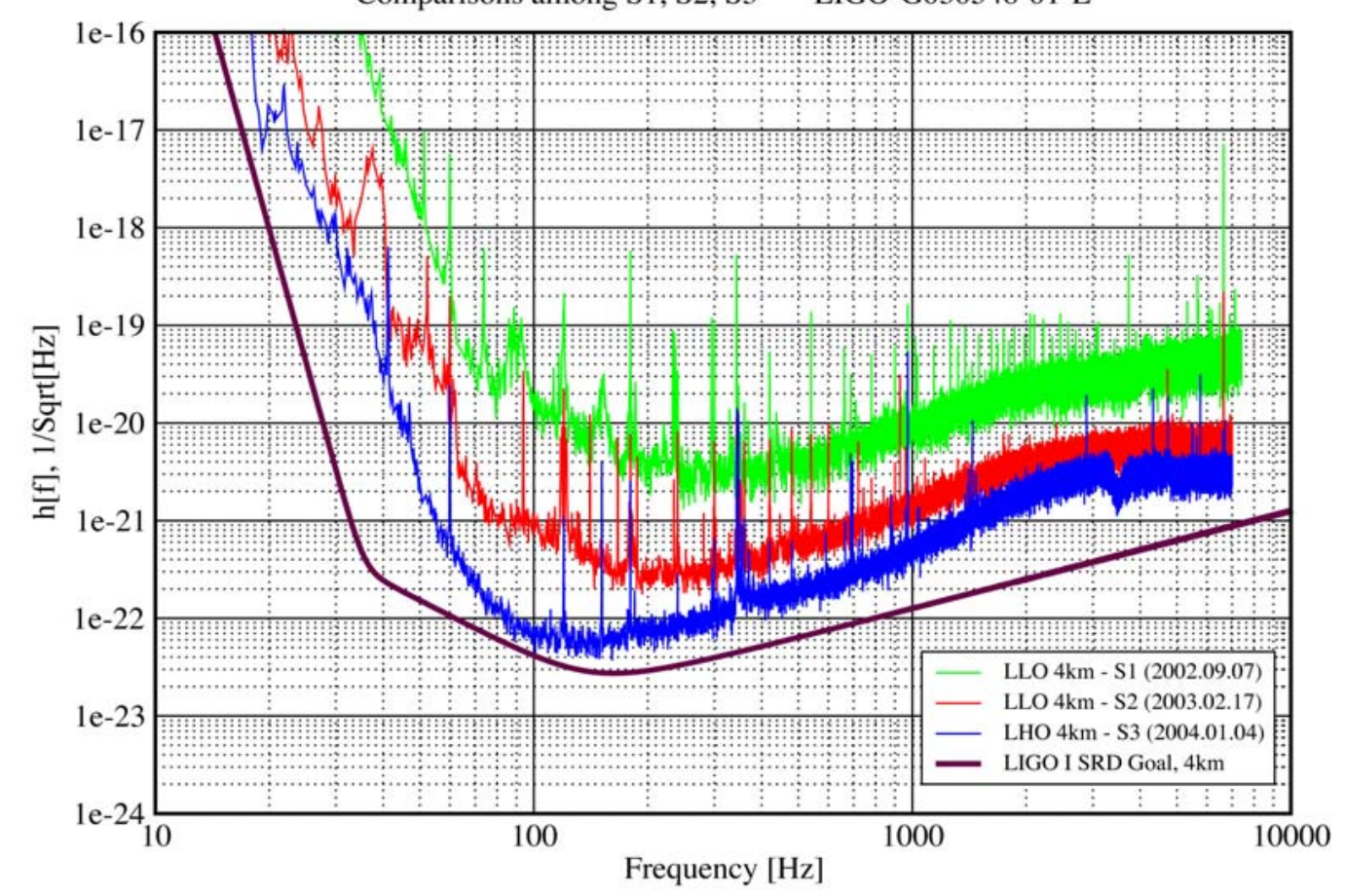

LIGO close to nominal sensitivity Science runs started

S1 (Sept 2002)

S2 (Feb 2003)

S3 (Jan 2004)

Virgo completed and being commissioned data taking in 2005 


\section{Chronology of stellar collapse}

- Core collapse

$\mathrm{p} \mathrm{e}^{-} \rightarrow \mathrm{n} v_{\mathrm{e}}$

neutronization

- supernuclear densities: ' $v$ sphere inside core ( $v$ trapped)

- Shock wave bounce propagating from deep inside core

$\Rightarrow \quad$ GW burst within a few ms

within $<1$ ms shock wave passes through $v$ sphere

$\Rightarrow \quad$ initial $v_{\mathrm{e}}$ burst (flash) a few ms

- High $\mathrm{T} \quad \mathrm{e}^{+} \mathrm{e}^{-} \rightarrow v_{\mathrm{i}} \bar{v}_{\mathrm{i}} \quad$ all $v$ types $(\mathrm{e}, \mu, \tau)$

shock turns on release of $v_{\mathrm{e}}$ and $v_{\mathrm{i}} v_{\mathrm{i}}$ pairs

$\Rightarrow \quad$ main $v$ burst $1-10 \mathrm{~s}$ long

- Accretion and explosion ( $v$ heating of shocked envelope)

$\Rightarrow \quad$ optical signal delayed by a few hrs 


\section{Simulation of neutrino burst}

- $\quad$ Model-independent properties

$99 \%$ of initial binding energy into $v$ 's (1-2\% in early flash) about $310^{53}$ erg released $\quad\left\langle\mathrm{E}_{v}>=10-20 \mathrm{MeV}\right.$

- Detailed numerical simulations

Mayle, Wilson, Barrows, Mezzacappa, Janka, .....
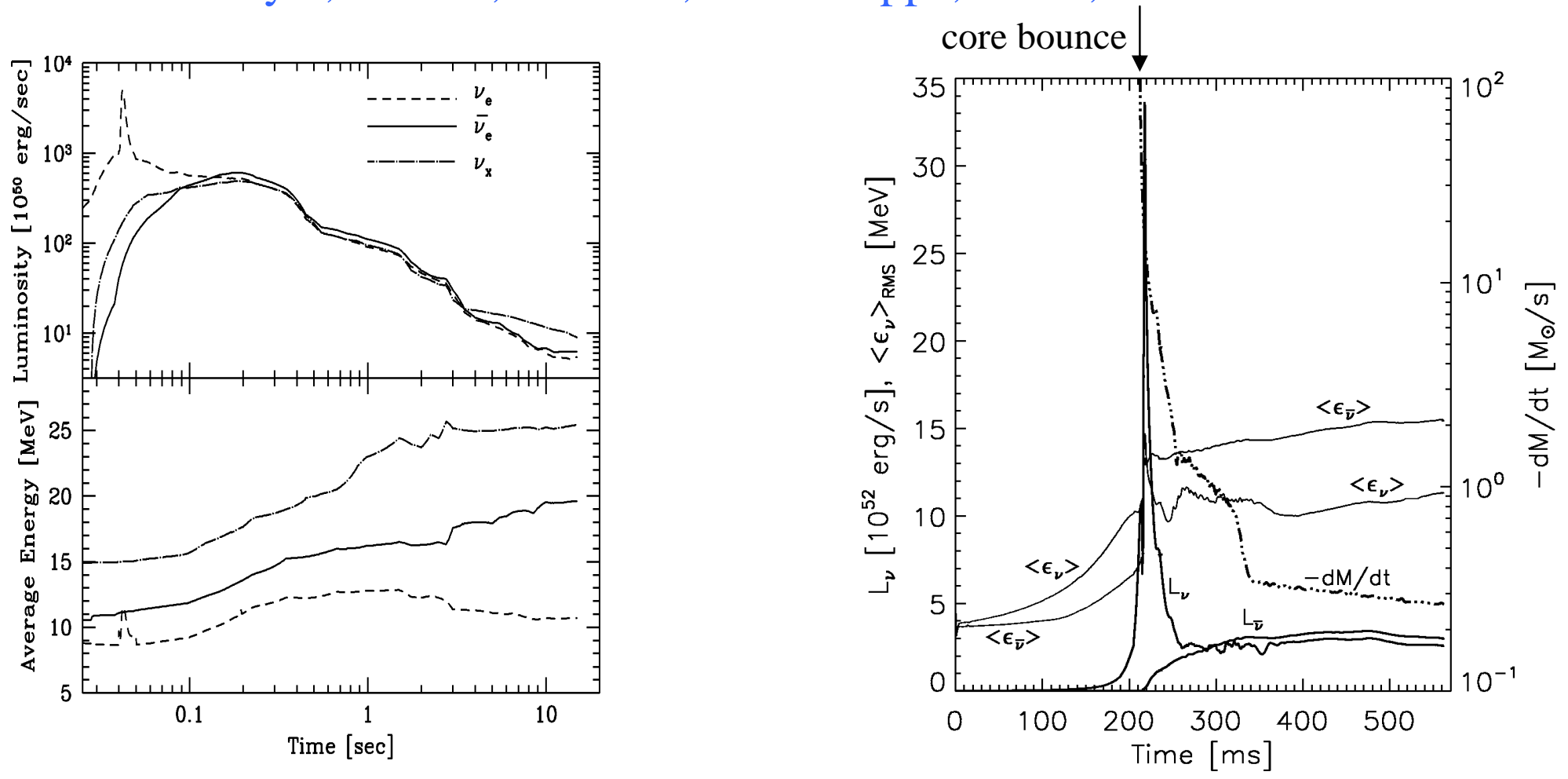

M. Davier

Neutrino 2004 


\section{Neutrino detection}

best operating detectors are water Cerenkov :

SuperK (32 kt) SNO(1 kt heavy water)

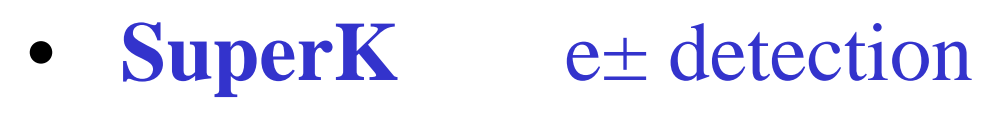
$v \mathrm{e}^{-} \rightarrow v \mathrm{e}^{-}$
directional
$\mathrm{E}_{\mathrm{e}}$ flat $0 \rightarrow \mathrm{E}_{v}$
$\overline{v_{\mathrm{e}}} \mathrm{p} \rightarrow \mathrm{e}^{+} \mathrm{n}$
non directional
$\mathrm{E}_{\mathrm{e}}=\mathrm{E}_{\mathrm{v}}-1.77 \mathrm{MeV}$

- SNO

$v_{\mathrm{e}} \mathrm{d} \rightarrow \mathrm{e}^{-} \mathrm{p} \mathrm{p} \quad$ non directional $\mathrm{E}_{\mathrm{e}}=\mathrm{E}_{\mathrm{v}}-1.44 \mathrm{MeV}$

$\overline{v_{\mathrm{e}}} \mathrm{d} \rightarrow \mathrm{e}^{+} \mathrm{n} \mathrm{n}$

4.03

$v_{\mathrm{i}} \mathrm{d} \rightarrow v_{\mathrm{i}} \mathrm{p}$ n

unique 


\section{Neutrino event rate (SN at $10 \mathrm{kpc}$ )}

SuperK

$v_{\text {e }}$

$\bar{v}_{\mathrm{e}}$

$v_{\mu}, v_{\tau}$

$v_{\mathrm{e}}$ flash

all
91

4300

(40)

12

4430
SNO

132

3

442

207

9

0.4

781

146 


\section{Supernova GW detection}

(1) Expected amplitude (simulations Zwerger-Müller 97)

LIGO-Virgo

$\mathrm{d}_{\text {mean }} \sim 30 \mathrm{kpc}$ threshold SNR $=5 \Rightarrow$ detection limited to our Galaxy

(2) Antenna patterns

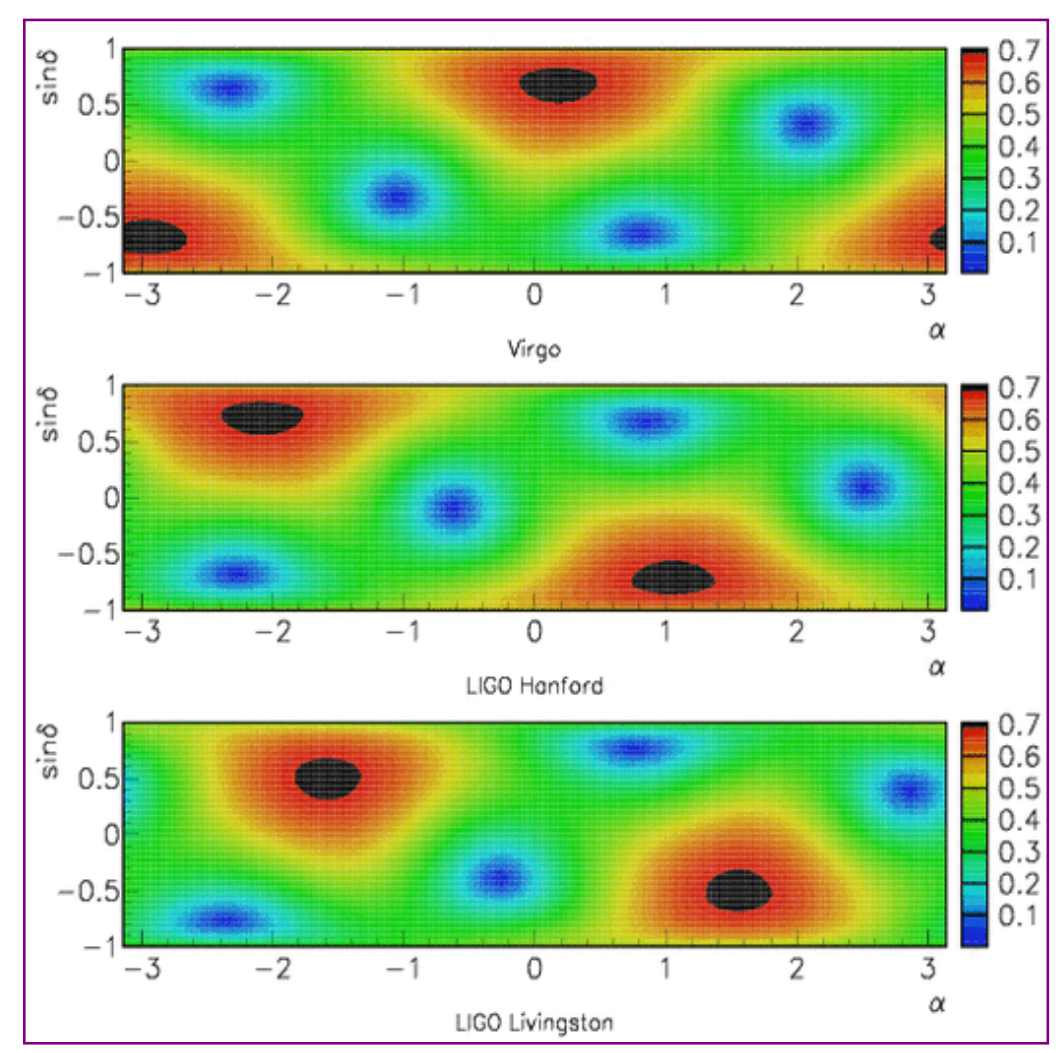

M. Davier
- Sky maps averaged over GW source polarization angle

- 2 LIGO interferometers mostly parallel

- Virgo nearly orthogonal to LIGO

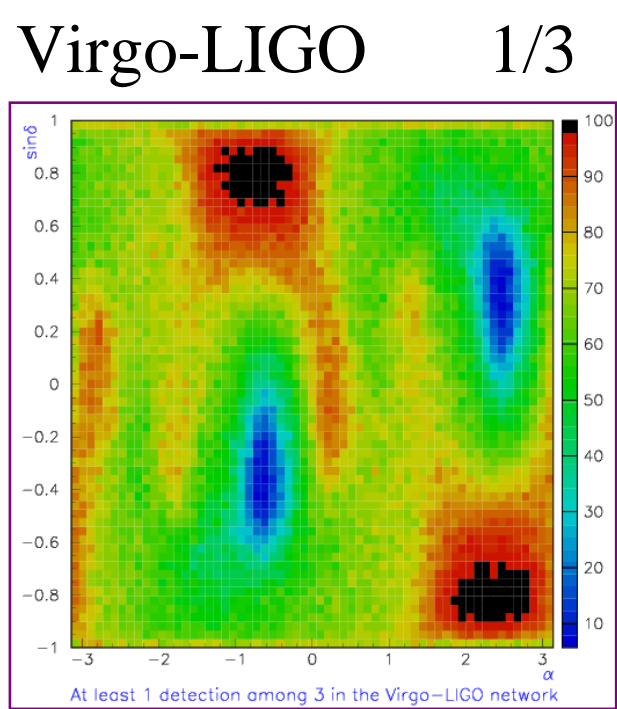

Neutrino 2004
Paris 11-16 June 2004

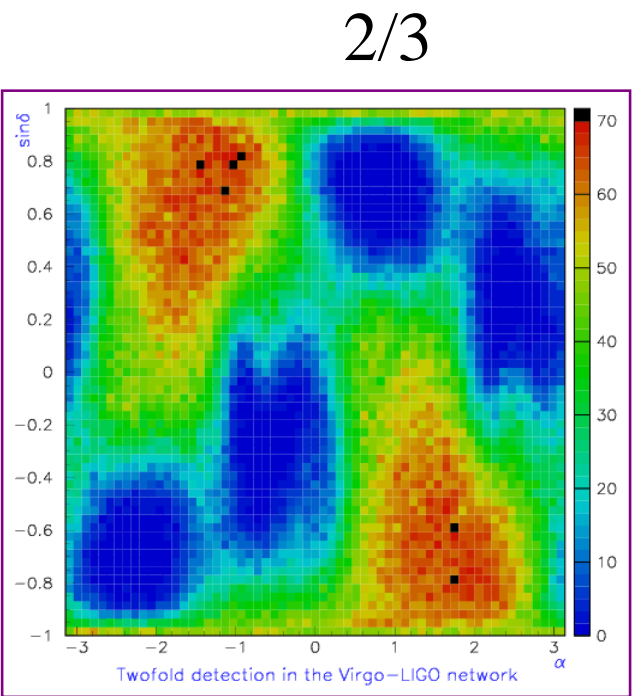

15 


\section{The next Galactic SN : GW-v coincidence strategy (1)}

- $v$ detectors

Arnaud 03

- several running detectors covering the Galaxy with an efficiency of 100\%

- false alarm rate negligible if at least 2 in coincidence

- direction to $\approx 5^{\circ} \quad$ (best precision from delayed optical observation)

- SNEWS network : alarm to astronomers + GW detectors within 30'

- GW interferometers

- relatively low threshold barely covers Galaxy, but false rate too high (assuming gaussian stationary noise, not realistic, so even worse)

- not suitable for sending alarms

- very important to react on $v$ alarms (discovery of GW from SN collapse)

- at least 2 antennas with complementary beam patterns needed for sky coverage, at least 3 to perform coincidences at reasonable efficiency 


\section{GW- $v$ coincidence strategy (2)}

loose coincidence strategy: correlate GW signals in several antennas without directional information (time window \pm $50 \mathrm{~ms}$, maximum time delay between antennas)

tight coincidence strategy: knowing source direction (from $v$ or optical), time window can be reduced to $\approx 10 \mathrm{~ms}$

coherent analysis : knowing source direction, outputs of all interferometers can be summed with weights $\propto$ beam pattern functions, only one threshold on sum, tight coincidence applied with neutrinos

Two goals:

- claim the discovery of GW emission in the SN collapse : require $10^{-4}$ accidental coincidence probability in $10 \mathrm{~ms}$ window

- study GW signal in coincidence with neutrinos : $10^{-2}$ enough 


\section{GW-v coincidence strategy (3)}

\section{LIGO - Virgo network}

Arnaud 03

Detection Probability in Coherent Analysis

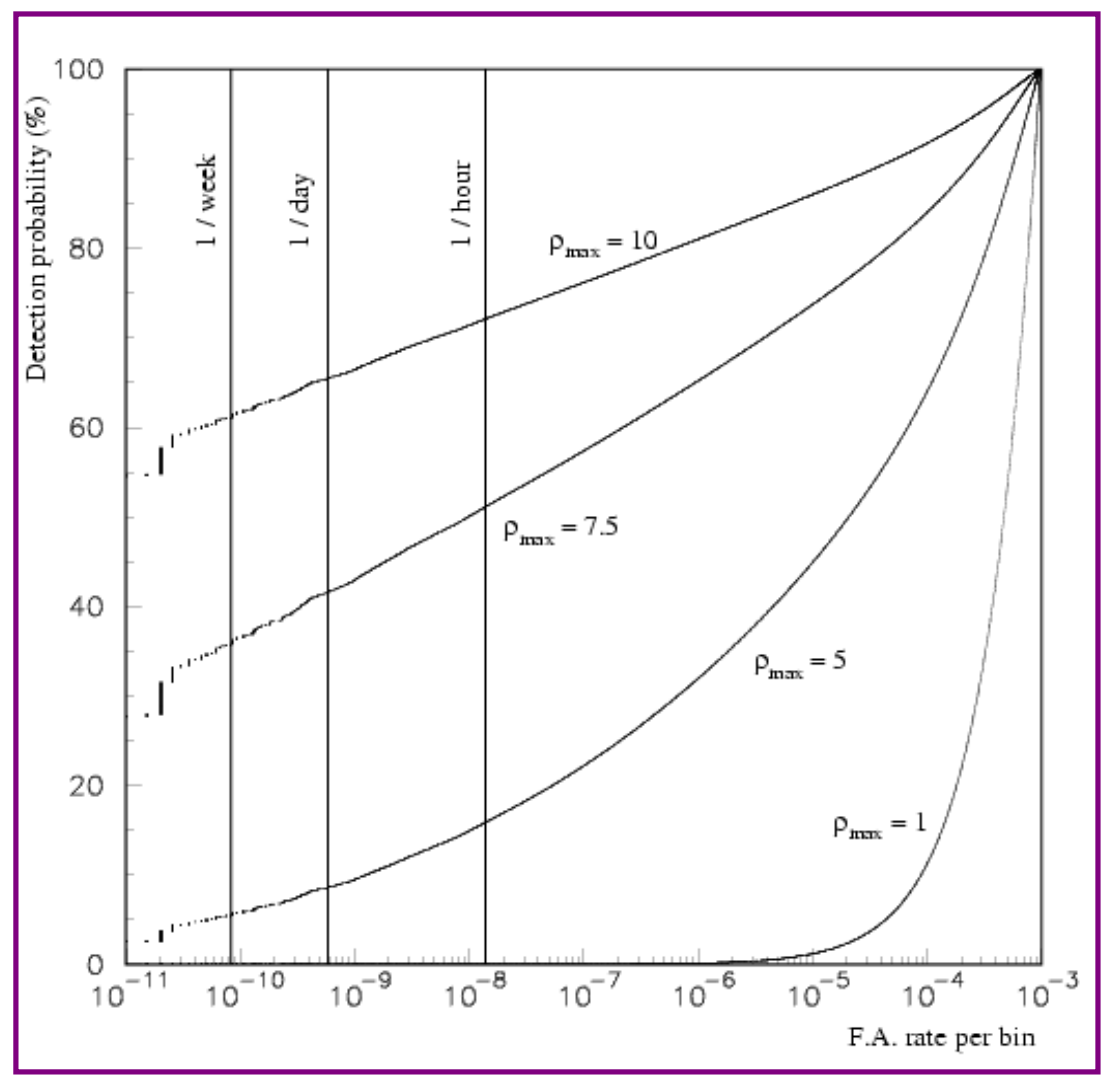

Accidental coincidence in $10 \mathrm{~ms}$

$\begin{array}{lcc}\text { Efficiency (\%) } & 10^{-4} & 10^{-2} \\ \text { Coincidence 2/3 } & 55 & 66 \\ \text { OR 1/3 } & 71 & 85 \\ \text { Coherent } & 80 & 91\end{array}$

$\Rightarrow$ Coherent analysis provides best efficiency for SN GW confirmation

False Alarm rate in sampling bin $(20 \mathrm{kHz})$ 


\section{GW/neutrino timing}

- SYST: GW peak time / bounce

$(0.1 \pm 0.4) \mathrm{ms} \quad$ Zweiger-Muller 97

- SYST: $v_{\mathrm{e}}$ flash / bounce

(3.5 \pm 0.5$) \mathrm{ms}$ simulations

- STAT: GW peak time accuracy $<0.5$ ms depends on filtering algorithm

- STAT: $v_{\mathrm{e}}$ flash accuracy $=\sigma_{\text {flash }} / \sqrt{\mathrm{N}_{\text {events }}}$

with $\quad \sigma_{\text {flash }}=(2.3 \pm 0.3) \mathrm{ms}$

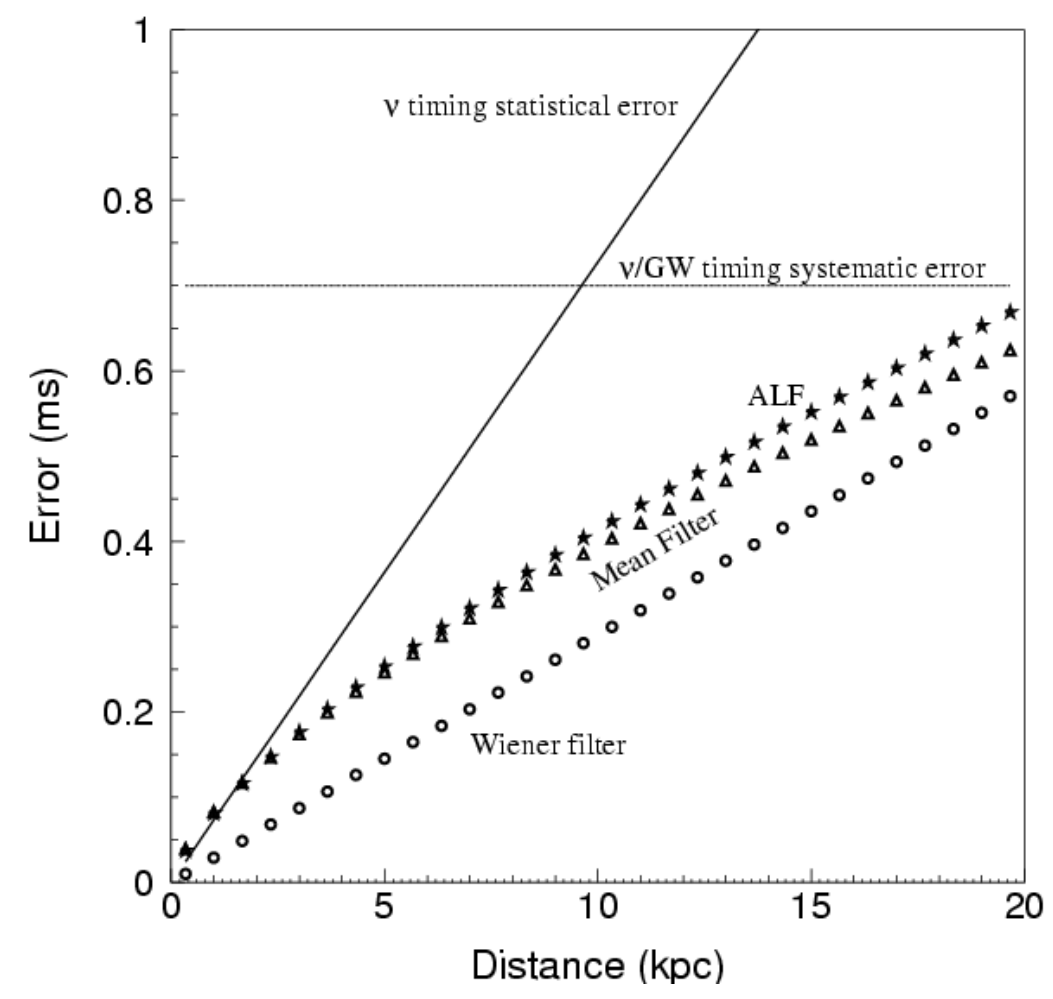

Arnaud 02, 03

to reduce systematic uncertainty joint simulations needed 


\section{GW/neutrino delay}

Pakvasa 72, Fargion 81, Arnaud 02

timing between the GW peak and the $v_{\mathrm{e}}$ flash

$\Delta \mathrm{t}_{v, \mathrm{GW}}=\Delta \mathrm{t}_{\text {prop }}+\Delta \mathrm{t}_{v \text {,bounce }}+\Delta \mathrm{t}_{\mathrm{GW} \text {, bounce }}$

$$
\begin{aligned}
\Delta \mathrm{t}_{\text {prop }} & =(\mathrm{L} / 2)\left(\mathrm{m}_{v} / \mathrm{E}_{v}\right)^{2} \\
& =5.2 \mathrm{~ms}(\mathrm{~L} / 10 \mathrm{kpc})\left(\mathrm{m}_{v} / 1 \mathrm{eV}\right)^{2}\left(10 \mathrm{MeV} / \mathrm{E}_{v}\right)^{2}
\end{aligned}
$$

- yields $\delta \mathrm{m}_{v}^{2} \propto \Delta \mathrm{t} / \mathrm{L} \approx$ constant

- accuracy of $\approx 1$ ms gives sensitivity to neutrino masses $<1 \mathrm{eV}$

- direct and absolute measurement

- if $v_{\mathrm{e}}$ mass obtained from other exp. to a precision $<0.5 \mathrm{eV}$, then $\mathrm{GW} / v_{\mathrm{e}}$ timing provides unique information on bounce dynamics 


\section{Simulating the experiment}

(a) SNO $v_{e} d$ CC
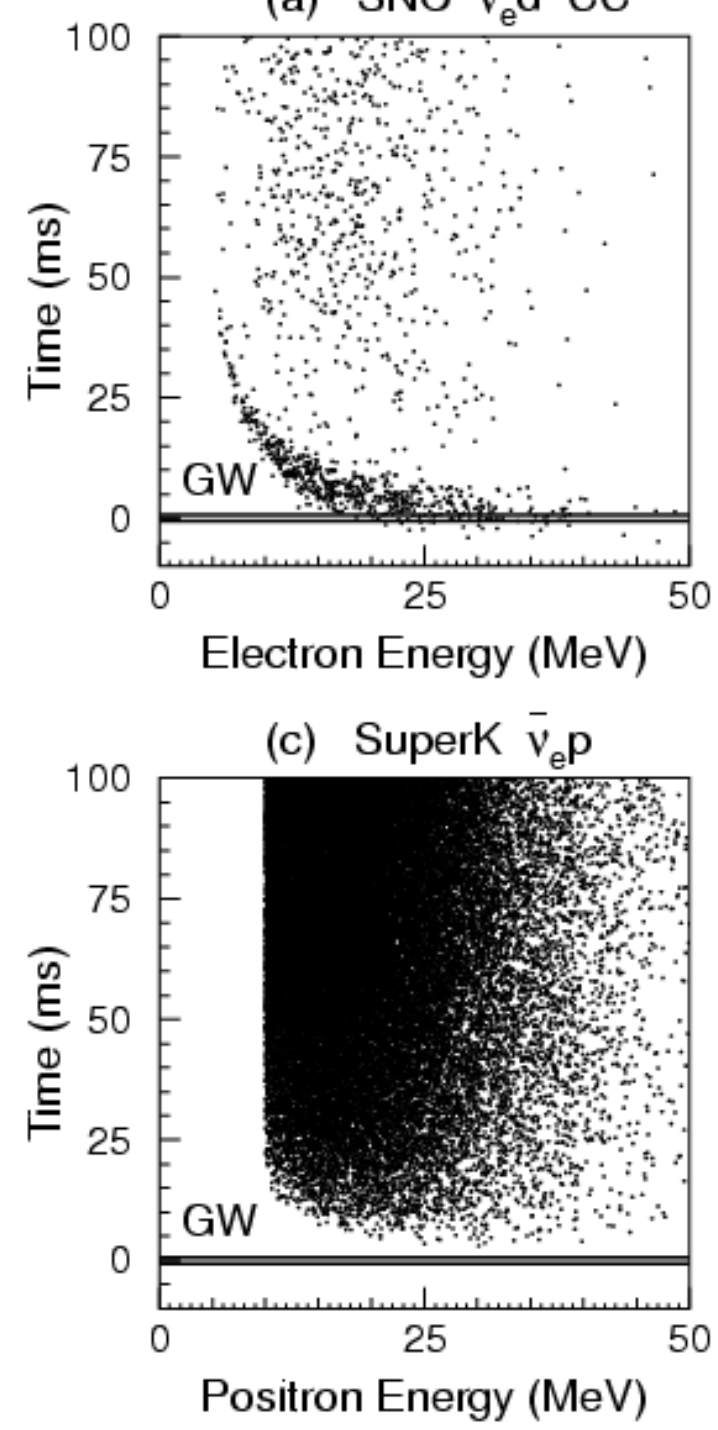

(b) SuperK $v_{\mathrm{e}} \mathrm{e}$

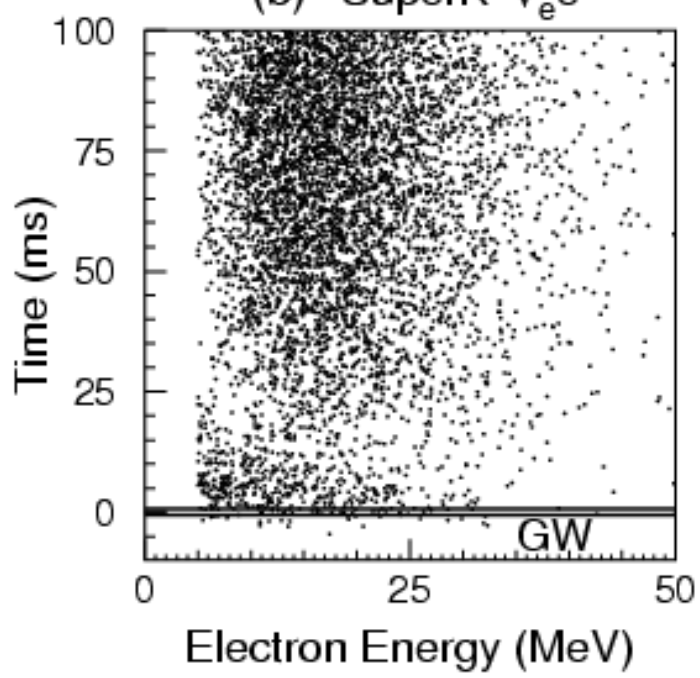

(d) SNO vd NC

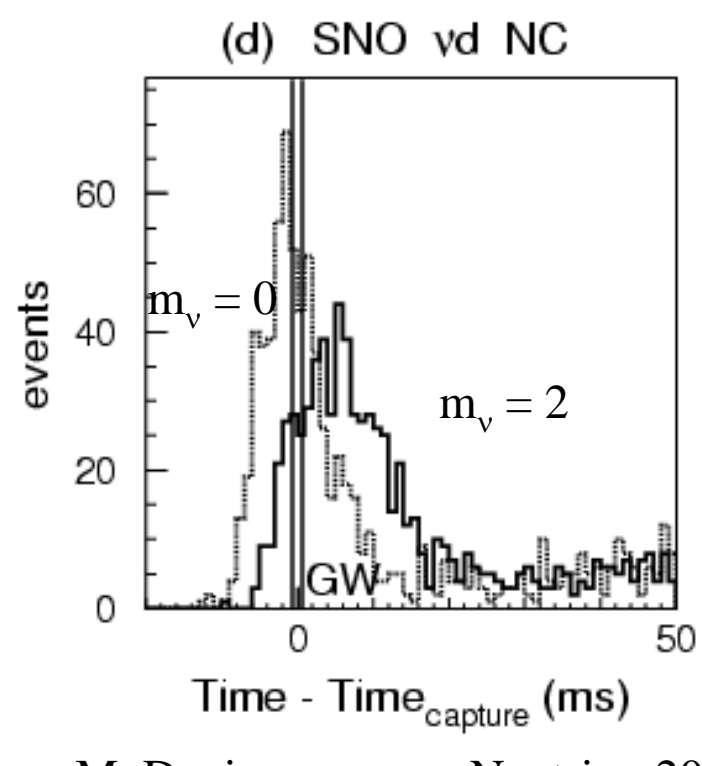

M. Davier Neutrino 2004

Paris 11-16 June 2004
SN collapse at $10 \mathrm{kpc}$ statistics $\mathrm{x} 100$

$$
\mathrm{m}_{\mathrm{v}}=2 \mathrm{eV}
$$

Arnaud 02 


\section{Expected results}

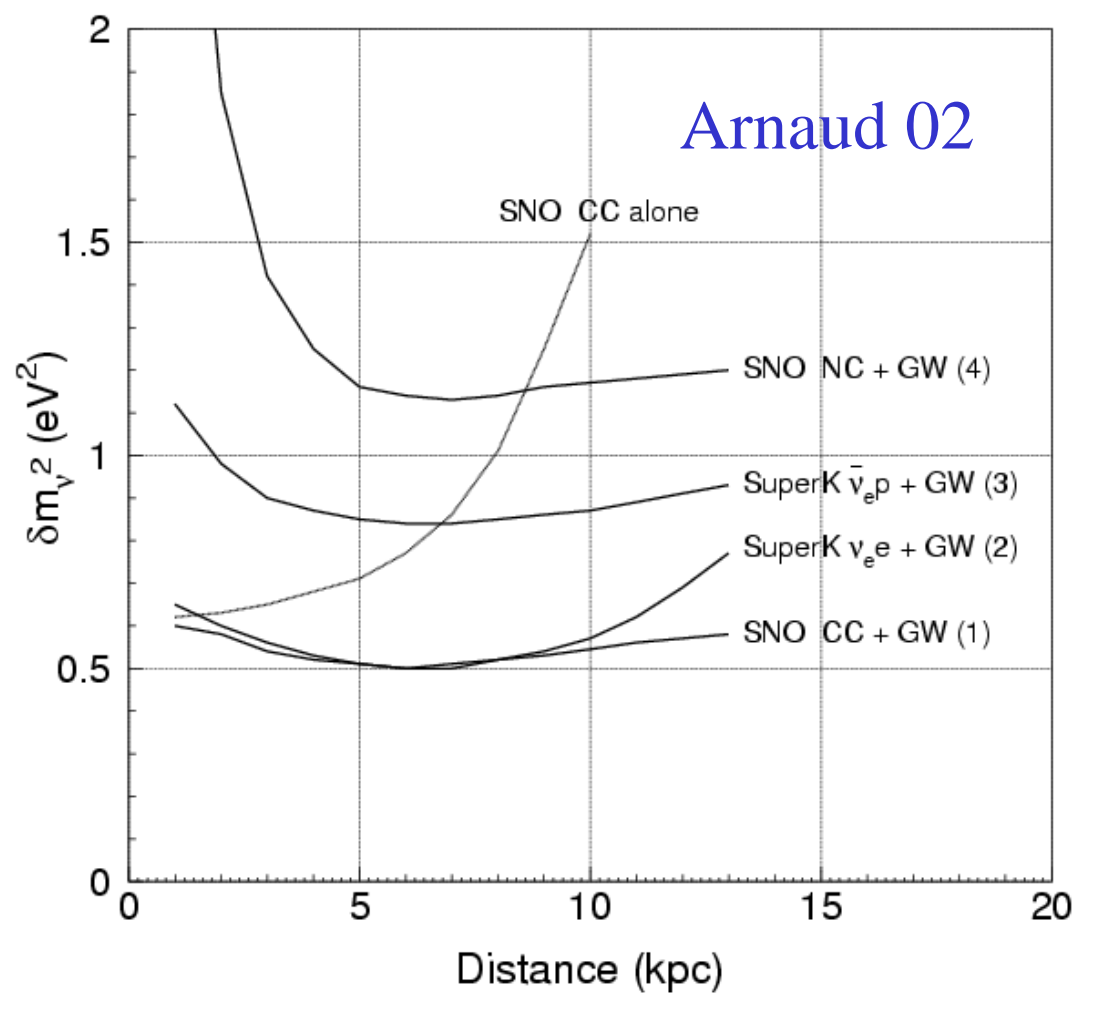

-methods $(1,2)$ with $\mathrm{P}_{\mathrm{e}}=0.5$

- method (4) when $\mathrm{P}_{\mathrm{e}}=0$

- method (3) whatever $\mathrm{P}_{\mathrm{e}}$
- results take into account neutrino oscillations (Dighe 00)

- relevant parameter: $v_{\mathrm{e}}$ survival probability $\mathrm{P}_{\mathrm{e}}$

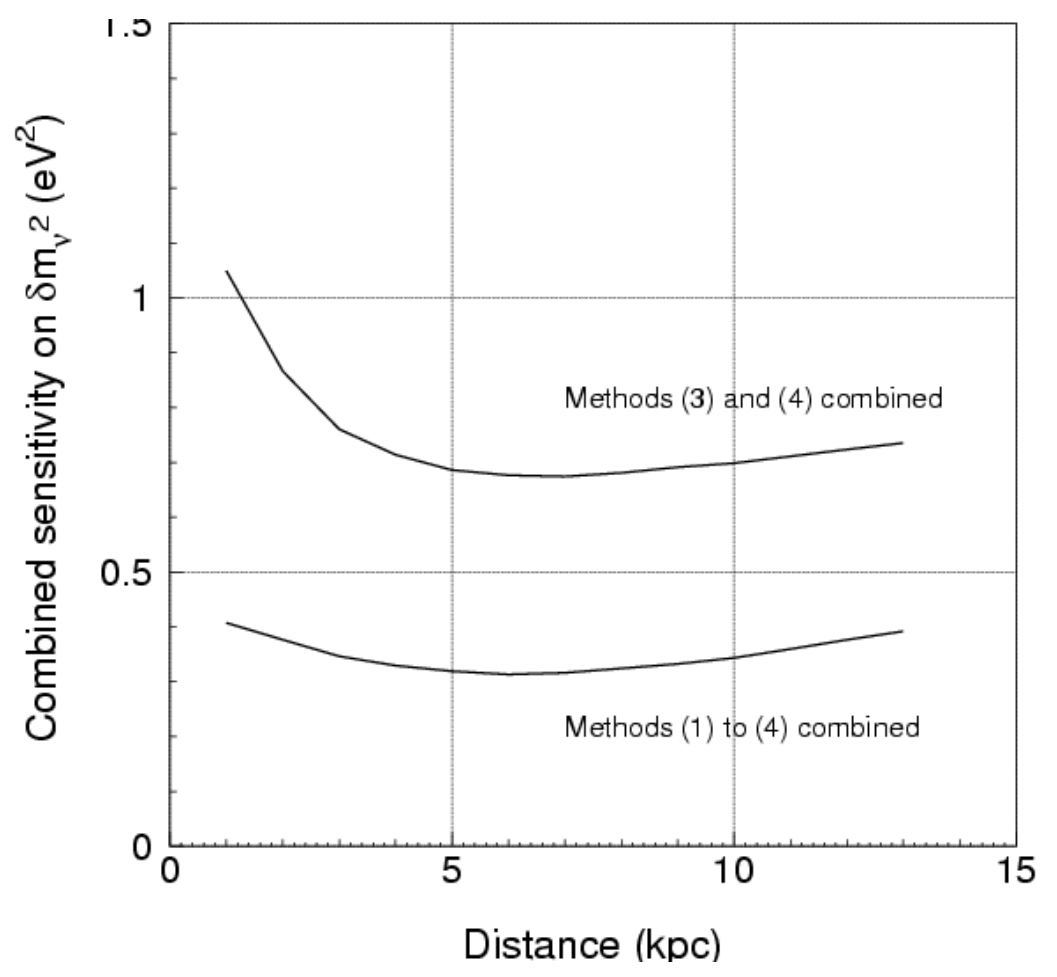

Neutrino 2004

22 


\section{Supernova physics (1)}

neutrino detection : time and energy spectra for $v_{\mathrm{e}}$ and $\bar{v}_{\mathrm{e}}$ time spectrum for $v_{\mu, \tau}$ luminosity (distance)

GW detection : $\quad$ timing (bounce) amplitude

timing of neutrino pulses / bounce to better than $1 \mathrm{~ms}$ if $v$ mass known or $<0.5 \mathrm{eV}$

learn about size of neutrinosphere (core opacity) and shock wave propagation velocity 


\section{Supernova physics (2)}

an interesting possibility : inner core collapse + accretion from outer mantle $\Rightarrow$ delayed Back Hole formation $\approx 0.5 \mathrm{~s}$

abrupt cutoff in neutrino time spectrum $\approx 0.5 \mathrm{~ms}$

could be used as a timing signal

to observe late neutrinos, but mass sensitivity limited to $1.8 \mathrm{eV}$

(Beacom 2000)

to search for $\mathrm{BH}$ ringdown signal in GW antennas : could run with relatively low threshold thanks to excellent timing, matched filtering (damped sines)

observations of a sharp cutoff in the neutrino time spectrum and a synchronized GW ringdown signal would constitute a smoking gun evidence for $\mathrm{BH}$ 


\section{Conclusions (1)}

- Complementary information on astrophysical phenomena is vital

- So far only used extensively with EM signals from radio to $\gamma$-rays (ex. GRBs)

- SN 1987a : extra-solar $v$ signal for the first time

- Study of the most violent events (collapses, mergers) will benefit enormously from the availability of $\gamma$, UHE cosmic rays, $v$ and GW detectors available and under construction

- Multiwavelength approach to cover a broad range of phenomena:

EM to-day's astrophysics

$v$ from $5 \mathrm{MeV}$ to $1000 \mathrm{TeV}$

GW Ligo-Virgo $10 \mathrm{~Hz}-10 \mathrm{kHz} \quad$ LISA $0.1-100 \mathrm{mHz}$

- Rates are small : need for large instruments

- Important to narrow the range of astrophysical interpretations 


\section{Conclusions (2)}

- A single Galactic SN event seen in coincidence in GW and $v$ detectors would bring unique information.

- Sky coverage requires OR-ing several antennas with complementary beam patterns.

- LIGO-Virgo network will be $80 \%$ efficient to discover GW emission by a SN seen by $v$ detectors with an accidental coincidence probability of $10^{-4}$.

- Precise GW/v timing can be achieved at better than $1 \mathrm{~ms}$.

- Absolute neutrino masses can be investigated below the present lower limit of $2 \mathrm{eV}$ down to $0.6-0.8 \mathrm{eV}$ in a direct way.

- When $v$ masses are known from other methods or found to be smaller than $0.5 \mathrm{eV}$, relative $\mathrm{GW} / v$ timing provides a new tool to investigate $\mathrm{SN}$ physics.

- If the SN eventually collapses into a BH, a GW/v coincidence analysis can prove the $\mathrm{BH}$ formation. 